\title{
Partida de reator anaeróbio compartimentado em série com um reator anaeróbio de manta de lodo, utilizando parâmetros de sedimentabilidade para formação da biomassa
}

Start-up of anaerobic baffled reactor in series with upflow anaerobic sludge blanket reactor reactors, using parameters of sedimentation for formation of sludge

\section{Cristine Serafine Neves', Claudio Milton Montenegro Campos²,} Erlon Lopes Pereira ${ }^{3}$, Regina Batista Vilas Boas ${ }^{4}$

\section{RESUMO}

Este estudo objetivou avaliar a formação da biomassa de boa qualidade tanto no reator anaeróbio compartimentado (RAC) quanto no reator anaeróbio de manta de lodo (UASB), aplicando parâmetros adequados à sedimentação dos sólidos sedimentáveis (SS) presentes na água residuária de suinocultura (ARS). O RAC e o UASB operaram em série com os tempos de detenção hidráulica (TDHs) de 15 e 9h, respectivamente, correspondendo a cargas orgânicas volumétricas (COVs) médias de 53,8 e 36,2 kg.m³. $\mathrm{d}^{-1}$ em termos de $\mathrm{DQO}^{\mathrm{T}}$ respectivamente. As eficiências médias de remoção de $\mathrm{DBO}_{5}^{20^{\circ} \mathrm{C}}$, durante o processo de partida e formação da biomassa, foram: 68\% para RAC e 55\% para UASB, e em termos de DQO: 78\% para RAC e 70\% para UASB, respectivamente. Os autores concluíram queé possível, mediante os parâmetros de sedimentação adotados, formar uma biomassa peletizada, mesmo operando o sistema com altas cargas orgânicas.

Palavras-chave: água residuária; reator anaeróbio compartimentado; eficiência; reator anaeróbio de manta de lodo e fluxo ascendente; água residuária de suinocultura.

\begin{abstract}
This study aimed to evaluate anaerobic biomass formation in anaerobic baffled reactor (ABR) and sequentialy in upflow anaerobic sludge blanket (UASB) reactor, using the solids liable to sedimentation present in swine wastewater (SW). The ABR and UASB reactors operated in series with hydraulic retention times (HRT) of 15 and 9. The average volumetric organic loading rates (VOLR) was about 53.8, and $36.2 \mathrm{~kg} \cdot \mathrm{m}^{-3} \mathrm{~d}^{-1}$ in terms of total COD, respectively. The average removal efficiencies, during the starting up process and biomass formation concernig $\mathrm{BOD}_{5}{ }^{20^{\circ} \mathrm{C}}$ about 68\% (ABR) and 55\% (UASB), and in terms of COD about 78\% (ABR) and 70\% (UASB), respectively. The authors concluded that it is possible, using the sedimentation parameters adopted, to form a granulated biomass, even operating with high organic loadings
\end{abstract}

Keywords: wastewater; anaerobic baffled reactor; efficiency; up flow anaerobic sludge blanket; swine wastewater.

\section{INTRODUÇÃO}

Tendo o Brasil clima favorável e disponibilidade de área com elevado potencial para expansão da suinocultura, objetivando aumentar a produção e a exportação de carne, deve-se buscar uma forma de manejar adequadamente a água residuária de suinocultura (ARS) gerada nesse sistema agroindustrial. Atualmente, o maior desafio para o setor de pesquisas agroambientais do país é garantir o aumento da produção, sem prejuízo da qualidade ambiental (PEREIRA et al., 2014; 2015).

O reator anaeróbio compartimentado (RAC) consiste em uma unidade com várias chicanas verticais que forçam o afluente a se

'Engenheira Química. Mestre em Engenharia Agrícola pela Universidade Federal de Lavras (UFLA) - Lavras (MG), Brasil.

${ }^{2}$ Graduado em Engenharia Civil com Ênfase em Estruturas pela Universidade Federal de Minas Gerais (UFMG). Especializado em Saneamento e Recursos Hídricos pela UFMG. Mestre em Saneamento, Meio Ambiente e Recursos Hídricos pela UFMG. Doutor em Environmental Engineering pela University of Newcastle Upon Tyne - United Kingdom. Professor Associado IV da UFLA - Lavras (MG), Brasil.

${ }^{3}$ Escola de Engenharia de Lorena da Universidade de São Paulo (EEL-USP). Engenheiro Agrícola pela UFLA. Mestre em Biotecnologia Industrial pela USP. Doutorando em Engenharia Agrícola na Universidade Federal de Viçosa (UFVA) - Viçosa (MG), Brasil.

${ }^{4}$ Graduada em Química. Mestre em Engenharia Agrícola pela UFLA - Lavras (MG), Brasil.

Endereço para correspondência: Erlon Lopes Pereira - Rua Tabelião Machado, 108, Apto. 101 - Lourdes - $36570-000$ - Viçosa (MG), Brasil - E-mail: erlonlopes@gmail.com Recebido: 18/06/13 - Aceito: 24/O2/15 - Reg. ABES: 119235 
movimentar por densas mantas compostas de populações bacterianas. Esse processo permite um maior contato entre a ARS e os microrganismos, conferindo maior eficiência na degradação da matéria orgânica particulada e solúvel, além de dificultar a perda de sólidos por arrastamento e diminuir a incidência de choques orgânicos e hidráulicos (PEREIRA; CAMPOS; MOTERANI, 2010b; MOTTERAN; PEREIRA; CAMPOS, 2013b). Outra significativa vantagem do RAC é a capacidade de hidrólise e acidificação, tornando o efluente adequado para o processo metanogênico (VAN HAANDEL; KATO; CAVALCANTI, 2006; PEREIRA et al., 2011).

O reator anaeróbio de manta de lodo e fluxo ascendente (UASB) consiste em uma unidade que possui no topo um decantador precedido por separador trifásico que favorece a separação entre biogás, biomassa e efluente. As características construtivas do UASB facilitam a coleta do biogás gerado (ZEEUW, 1984; PEREIRA; CAMPOS; MOTERANI, 2009; PEREIRA; CAMPOS; MOTERANI, 2010a; CAMPOS; PRADO; PEREIRA, 2014). Devido as suas características hidrodinâmicas e operacionais, o UASB tem sido cada vez mais utilizado, principalmente em processos metanogênicos, apresentando elevada eficiência no tratamento de águas residuárias com predominância de matéria orgânica solúvel. Todavia, assim como outros reatores anaeróbios de alta taxa, apresenta limitações para o tratamento de águas residuárias com elevadas concentrações de sólidos suspensos (VAN HAANDEL \& LETTINGA, 1994; VAN HAANDEL; KATO; CAVALCANTI, 2006; CAMPOS; PRADO; PEREIRA, 2014).

Segundo Kim et al. (2009), uma das grandes dificuldades no tratamento de ARS é a alta concentração de sólidos suspensos prejudiciais à hidrólise. $\mathrm{O}$ uso do processo anaeróbio em dois estágios, com a hidrólise parcial da matéria orgânica particulada (no primeiro reator) e a conversão (no segundo reator) dos compostos solúveis formados no primeiro reator, pode atenuar esse problema (OLIVEIRA \& SANTANA, 2011; PEREIRA et al., 2011; PEREIRA; CAMPOS; MOTERANI, 2013).

Durante o processo de tratamento de efluentes há duas fases distintas e importantes: a partida (start-up) e o estado estacionário (steady-state). Para realizar a partida, geralmente inocula-se o reator com biomassa aclimatizada proveniente de outros reatores, normalmente obtida em estações de tratamento de esgoto em centros urbanos (METCALF \& EDDY, 2003). A aquisição dessa biomassa exige transporte e condicionamento térmico, tornando a inoculação um processo trabalhoso e oneroso. Com base nisso, muitos suinocultores não teriam condições de adquirir lodo para inoculação dos reatores utilizados no tratamento da ARS devido à distância, ao preço e à dificuldade de aquisição. Isso, de certa forma, se torna um empecilho na propagação do saneamento em áreas rurais. Portanto, este trabalho buscou dar alternativas aos suinocultores com relação à utilização de processos anaeróbios sem a necessidade de inoculação, apresentando dados e informações relevantes para o sucesso e a expansão dos processos anaeróbios no meio rural.

Motteran, Pereira e Campos (2013a) utilizaram em escala piloto um tanque de acidificação e equalização (TAE) no tratamento primário de ARS e observaram que, devido à alta quantidade de sólidos suspensos e decantáveis presentes na ARS e das condições anaeróbias submetidas ao TAE, houve formação natural de uma biomassa ativa. O mesmo poderia acontecer com reatores anaeróbios durante a partida, facilitando para o suinocultor a obtenção da biomassa e, consequentemente, estimulando o uso de reatores anaeróbios em suinoculturas e solucionando os problemas do impacto ambiental causado pela ARS não tratada.

Este trabalho objetivou avaliar o processo de partida em RAC e UASB em escala piloto, operando em série, sem a inoculação de biomassa, uma vez que a geração de biomassa nos reatores foi por meio da decantação dos sólidos sedimentáveis (SS) presentes na própria ARS.

\section{MATERIAL E MÉTODOS}

\section{Aparato experimental}

A estação piloto de tratamento de efluentes de suinocultura (EPTES) foi construída no setor de suinocultura do Departamento de Zootecnia (DZO) da Universidade Federal de Lavras (UFLA). As coordenadas geográficas locais foram: latitude $21^{\circ} 14^{\prime} \mathrm{S}$, longitude $45^{\circ} 00^{\prime} \mathrm{W}$ e $918 \mathrm{~m}$ de altitude. O clima, segundo a classificação climática de Köppen, é Cwa, com inverno seco e verão chuvoso.

A ARS utilizada continha fezes, urina, restos de ração, além da água de lavagem das baias. A EPTES foi composta por unidades de tratamento preliminar (caixa de retenção de sólidos, interligada em série com uma peneira estática), unidade de tratamento primário (TAE), unidades de tratamento secundário (RAC e UASB) e unidade de tratamento terciário (fotoreatores com radiação ultravioleta artificial para desinfecção da ARS). As unidades de tratamento estavam interligadas em série na ordem mencionada.

O RAC estudado foi composto por 3 câmaras C1, C2 e C3, com respectivos volumes líquidos: 1,72, 2,12 e 2,24 $\mathrm{m}^{3}$, cujas áreas correspondentes a cada compartimento foram de 0,638 , 0,787 e $0,832 \mathrm{~m}^{2}$. Esse reator foi construído de tijolos (assentados com argamassa extraforte e revestidos internamente com manta asfáltica) e posteriormente impermeabilizado com fibra de vidro, aumentando sua capacidade de estanqueidade. O fluxo ascendente em cada compartimento era equalizado por meio de calhas niveladas, construídas em fibra de vidro com vários vertedouros triangulares (Thompson), proporcionando um fluxo ascendente 
homogêneo em cada um dos compartimentos. O RAC possuía em sua parte superior três compartimentos em série, onde a produção de biogás era acumulada de maneira independente e a biomassa de cada compartimento apresentava características distintas. Maiores detalhes do projeto e a descrição física detalhada do RAC foram descritos por Pereira, Campos e Moterani (2010b) e Motteran, Pereira e Campos (2013b).

Após o efluente líquido passar ascendentemente, em cada uma das três câmaras, a ARS efluente do RAC foi encaminhada gravitacionalmente para o UASB. Essa unidade possuía volume útil de 3.815 L, sendo construída de alvenaria, impermeabilizada com manta asfáltica e também revestida com fibra de vidro. Na parte superior foi instalado um separador trifásico construído com meia manilha de concreto vibrado, onde o biogás era coletado e conduzido, por intermédio de tubos de PVC de 12,5 mm de diâmetro, ao equalizador de pressão. O separador trifásico possuía dois defletores construídos lateralmente, situados na parte inferior, cuja função era separar, por meio do atrito causado pela turbulência do líquido e do biogás, o lodo ascendido do biogás agregado. Esse processo de separação foi fundamental, uma vez que o biogás era liberado e as partículas sólidas do lodo decantam tornando a manta de lodo, aumentando significativamente a eficiência. Maiores detalhes podem ser vistos em Pereira, Campos e Moterani (2009; 2010a).

\section{Partida do reator anaeróbio compartimentado e do reator anaeróbio de manta de lodo}

O RAC e o UASB foram enchidos com ARS proveniente do tratamento primário do TAE e deixados estagnados durante seis dias, para purgar qualquer quantidade de oxigênio presente no meio, em função da turbulência causada na alimentação das unidades. Durante o período de 6 dias, antes da partida, foram feitas 10 amostragens da ARS afluente do RAC e foi determinada a quantidade de SS em cone Imhoff, denominado índice volumétrico de lodo (IVL), com base no tempo de sedimentação de 30 minutos. Após a sedimentação, foi realizada análise dos sólidos totais, voláteis e fixos utilizando apenas os sólidos sedimentados. Os resultados médios para o IVL e sólidos totais foram de $25,5 \mathrm{~mL} . \mathrm{L}^{-1}$ e de $24.528 \mathrm{mg} . \mathrm{L}^{-1}$, respectivamente. Para o processo de partida por sedimentação, teve-se como pressuposto:

- o cone Imhoff agia como um pequeno reator, operado em bateladas, sendo que os SS equivaleriam à biomassa que ficaria retida por sedimentação;

- a altura do lodo acumulado no RAC deveria atingir o último amostrador, cujo registro estava a uma altura de $1,68 \mathrm{~m}$ da base, para determinação do volume máximo de lodo (VLR);
- a partida foi realizada em função do RAC, onde a sedimentação dos sólidos deveria se dar em maior quantidade. Os sólidos excedentes sedimentariam no UASB.

Com base nesses pressupostos foram calculados os parâmetros hidráulicos de sedimentabilidade para o RAC (Tabela 1) e para o UASB (Tabela 2), sendo que a marcha de cálculo de cada um dos parâmetros foi demonstrada pelas Equações 1 a 11.

$\mathrm{VLR}=\mathrm{C} \times \mathrm{L} \times \mathrm{H}$

ISL convertido: conversão volumétrica do ISL padrão para a unidade de volume do reator $\left(\mathrm{m}^{3}\right)$ calculado pela Equação 2. Sendo o ISL padrão a média de sólidos sedimentáveis das 10 amostras determinados no cone de Imhoff com $0,5 \mathrm{~h}$ de teste para cada amostra, resultando em 25,5 mL.L $\mathrm{L}^{-1}$.

$25,5 \mathrm{~mL} \cdot \mathrm{L}^{-1}=25,5 \times 10^{-3} \frac{\mathrm{L} \text { sólidos }}{\mathrm{L} \text { de reator }}=25,5 \times 10^{-3} \frac{\mathrm{m}^{3} \text { sólidos }}{\mathrm{m}^{3} \text { de reator }}$

Tabela 1 - Parâmetros de sedimentação aplicados ao reator anaeróbio compartimentado.

\begin{tabular}{l|c|c} 
Parâmetros & Resultados & Unidades \\
VLR & 4,48 & $\mathrm{~m}^{3}$ \\
\hline VT & 6,08 & $\mathrm{~m}^{3}$ \\
\hline ISL padrão & 25,5 & $\mathrm{~mL}$ de sólidos $\mathrm{mL}^{-1}$ de amostra \\
\hline ISL convertido & 25,5 & $\mathrm{~m}^{3}$ de sólidos $\mathrm{m}^{-3}$ de amostra \\
\hline ISL $_{\text {RAC }}$ & 0,74 & $\mathrm{~m}^{3}$ de sólidos $\mathrm{m}^{-3}$ de amostra \\
\hline TDH & $\mathrm{H}$ \\
\hline $\mathrm{Q}_{\text {RAC }}$ & 14,5 & $\mathrm{~L} \cdot \mathrm{s}^{-1}$ \\
\hline TAS & 0,12 & $\mathrm{~m}^{3} \cdot \mathrm{m}^{-3} \cdot \mathrm{d}^{-1}$ \\
\hline $\mathrm{VA}_{\text {RAC }}$ & 4,56 & $\mathrm{~m} \cdot \mathrm{h}^{-1}$ \\
\hline
\end{tabular}

ISL: índice de sólidos sedimentáveis da água residuária de suinocultura em cone Imhoff; TDH: tempo de detenção hidráulica; TAS: taxa de aplicação superficial; VA: velocidade ascensional da áqua residuária de suinocultura; RAC: reator anaeróbio compartimentado; $\mathrm{VT}_{\mathrm{RAC}}$ : volume total do reator anaeróbio compartimentado; VLR: volume de lodo no reator anaeróbio compartimentado; ARS: água residuária de suinocultura.

Tabela 2 - Parâmetros de sedimentação aplicados ao reator anaeróbio de manta de lodo com base nos parâmetros aplicados ao reator anaeróbio compartimentado.

\begin{tabular}{l|c|c} 
Parâmetro & Resultado & Unidade \\
\hline$V_{\text {UASB }}$ & 3,815 & $\mathrm{~m}^{3}$ \\
\hline$A_{\text {UASB }}$ & 1,783 & $\mathrm{~m}^{2}$ \\
\hline $\mathrm{Q}_{\text {UASB }}=\mathrm{Q}_{\text {RAC }}$ & 0,421 & $\mathrm{~m}^{3} \cdot \mathrm{h}^{-1}$ \\
\hline$T D H_{\text {UASB }}$ & 9,06 & $\mathrm{H}$ \\
\hline$C H V_{\text {UASB }}$ & 2,65 & $\mathrm{~m}^{3} \cdot \mathrm{m}^{-3} \cdot \mathrm{d}^{-1}$ \\
\hline TAS $_{\text {UASB }}$ & 5,67 & $\mathrm{~m}^{3} \cdot \mathrm{m}^{-2} \cdot \mathrm{d}^{-1}$ \\
\hline $\mathrm{VA}_{\text {UASB }}$ & 0,236 & $\mathrm{~m} \cdot \mathrm{h}^{-1}$ \\
\hline
\end{tabular}

$\mathrm{Q}_{\text {UASB: }}$ : vazão aplicada ao reator anaeróbio de manta de lodo; $\mathrm{TDH}_{\text {UASB: }}$ : tempo de detenção hidráulica; $\mathrm{CHV}_{\text {UASB: }}$ : carga hidráulica volumétrica; TAS UASB: taxa de aplicação

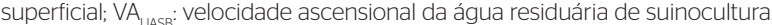




$$
\begin{aligned}
& \mathrm{ISL}_{\mathrm{RAC}}=\frac{\mathrm{VLR}_{\mathrm{RAC}}}{\mathrm{VT}_{\mathrm{RA}}} \\
& \mathrm{TDH}_{\mathrm{RAC}}=\frac{\mathrm{ISL}_{\text {RAC }} \times \mathrm{T}_{\mathrm{S}}}{\mathrm{ISL}_{\text {Convertido }}} \\
& \mathrm{Q}_{\mathrm{RAC}}=\frac{\mathrm{VT}_{\mathrm{RAC}}}{\mathrm{TDH}_{\mathrm{RAC}}} \\
& \mathrm{TAS}=\frac{\mathrm{Q}_{\mathrm{RAC}}}{\mathrm{A}_{\mathrm{RAC}}} \\
& \mathrm{V}=\frac{\mathrm{Q}_{\mathrm{RAC}}}{\mathrm{A}_{\mathrm{RAC}}}
\end{aligned}
$$

onde:

C: comprimento da base do RAC $(2,26 \mathrm{~m})$

L: largura da base do RAC (1,18 m)

$\mathrm{H}$ : altura da base até o último amostrador $(1,68 \mathrm{~m})$

VLR: volume de lodo requerido $\left(\mathrm{m}^{3}\right)$

$\mathrm{VT}_{\mathrm{RAC}}$ : volume total do RAC $\left(\mathrm{m}^{3}\right)$

$\mathrm{ISL}_{\mathrm{RAC}}$ : índice de sólidos suspensos da ARS calculado para as condições do RAC

$\mathrm{Q}_{\mathrm{RAC}}$ : vazão aplicada ao RAC necessária para que ocorra a sedimentação $\left(\mathrm{m}^{3} \cdot \mathrm{h}^{-1}\right)$

$\mathrm{TDH}_{\mathrm{RAC}}$ : tempo de detenção hidráulica para que ocorra a sedimentação no RAC (h)

$\mathrm{T}_{\mathrm{S}}$ : tempo de sedimentação utilizado no teste em cone de Imhoff (h)

TAS $_{\mathrm{RAC}}$ : taxa de aplicação superficial $\left(\mathrm{m}^{3} \cdot \mathrm{m}^{2} \cdot \mathrm{d}^{-1}\right)$
$\mathrm{VA}_{\mathrm{RAC}}$ : velocidade ascensional da ARS (m.h $\left.\mathrm{h}^{-1}\right)$

$\mathrm{A}_{\mathrm{RAC}}$ : área transversal do $\mathrm{RAC}\left(\mathrm{m}^{2}\right)$

$\mathrm{TDH}_{\mathrm{UASB}}=\frac{\mathrm{V}_{\mathrm{UASB}}}{\mathrm{Q}}$

$\mathrm{CHV}_{\text {UASB }}=\frac{1}{\mathrm{TDH}_{\text {UASB }}}$

$\mathrm{TAS}_{\mathrm{UASB}}=\frac{\mathrm{Q}}{\mathrm{A}_{\mathrm{UASB}}}$

$\mathrm{VA}_{\mathrm{UASB}}=\frac{\mathrm{Q}}{\mathrm{A}_{\mathrm{UASB}}}$

onde:

$\mathrm{TDH}_{\mathrm{UASB}}$ : tempo de detenção hidráulica aplicado ao UASB (h)

$\mathrm{V}_{\mathrm{UASB}}$ : volume total do UASB $\left(\mathrm{m}^{3}\right)$

$\mathrm{Q}_{\mathrm{UASB}}$ : vazão afluente ao UASB $\left(\mathrm{m}^{3} \cdot \mathrm{h}^{-1}\right)$

$\mathrm{CHV}_{\mathrm{UASB}}$ : carga hidráulica volumétrica aplicada ao UASB $\left(\mathrm{m}^{3} \cdot \mathrm{m}^{-3} \cdot \mathrm{h}^{-1}\right)$

TAS $_{\text {UASB }}$ : taxa de aplicação superficial submetida ao UASB $\left(\mathrm{m}^{3} \cdot \mathrm{m}^{-2} \cdot \mathrm{d}^{-1}\right)$

$\mathrm{VA}_{\mathrm{UASB}}$ : velocidade ascensional aplicada ao $\operatorname{UASB}\left(\mathrm{m} \cdot \mathrm{h}^{-1}\right)$

$\mathrm{A}_{\mathrm{UASB}}$ : área transversal do UASB $\left(\mathrm{m}^{2}\right)$

A partida do RAC e do UASB foi realizada sem inoculação e o

\begin{tabular}{|c|c|c|}
\hline Parâmetros físico-químicos & Frequência & Referência bibliográfica \\
\hline Temperatura e umidade & Diária & Leitura em termo-higrômetro \\
\hline $\mathrm{pH}$ & $2 \times$ semana & APHA; AWWA; WEF (2005) \\
\hline $\begin{array}{l}\text { Alcalinidade total (AT), alcalinidade intermediária (AI) e alcalinidade } \\
\text { parcial (AP) }\end{array}$ & Semanal & Ripley, Boyle e Converse. (1986) \\
\hline Condutividade elétrica (CE) & $2 \times$ semana & APHA; AWWA; WEF (2005) \\
\hline Acidez total (AcT) & Semanal & APHA; AWWA; WEF (2005) \\
\hline Demanda química de oxigênio total $\left(\mathrm{DQO}_{T}\right)$ e solúvel $\left(\mathrm{DQO}_{S}\right)$ & $2 \times$ semana & APHA; AWWA; WEF (2005) \\
\hline Demanda bioquímica de oxigênio total $\left.\mathrm{DBO}_{5}^{20^{\circ} \mathrm{C}} \mathrm{T}\right)$ e solúvel $\left(\mathrm{DBO}_{5}^{20^{\circ} \mathrm{C}} \mathrm{S}\right)$ & $2 \times$ semana & $\begin{array}{l}\text { APHA; AWWA; WEF (2005) e determinação de oxigênio } \\
\text { dissolvido pelo método de Wincley modificado }\end{array}$ \\
\hline Sólidos totais (ST), fixos (STF) e voláteis (STV) & $2 \times$ semana & APHA; AWWA; WEF (2005) \\
\hline Sólidos dissolvidos totais (SDT), fixos (SDF) e voláteis (SDV) & $2 \times$ semana & APHA; AWWA; WEF (2005) \\
\hline Nitrogênio total de Kjedahl (NTK) & Semanal & APHA; AWWA; WEF (2005) \\
\hline Fósforo total $\left(\mathrm{P}_{\mathrm{T}}\right)$ & Semanal & APHA; AWWA; WEF (2005) \\
\hline Óleos e graxas (O\&G) & Semanal & APHA; AWWA; WEF (2005) \\
\hline Sólidos sedimentáveis (SS) & $2 \times$ semana & APHA; AWWA; WEF (2005) \\
\hline
\end{tabular}
lodo foi formado por meio da sedimentação dos sólidos provenientes da ARS durante três meses. As amostragens para realização das análises físico-químicas (Tabela 3) foram realizadas de forma composta

Tabela 3 - Análises físico-químicas, frequências e metodologias utilizadas. 
ao longo do dia em três pontos: afluente ao RAC (A-RAC), efluente do RAC e ao mesmo tempo afluente ao UASB (E-RAC) e efluente ao UASB (E-UASB).

Para análise do perfil do lodo no RAC e no UASB, foram realizadas dez coletas ao longo da altura dos amostradores, sendo a primeira coletada sete dias após o início da partida do sistema, e as outras nove coletas realizadas semanalmente. A última realizada 100 dias após a partida.

As análises físico-químicas da ARS e do lodo foram realizadas no Laboratório de Análises de Água do Departamento de Engenharia da Universidade Federal de Lavras (LAADEG-UFLA). Os parâmetros físico-químicos analisados e suas respectivas frequências e metodologias estão apresentados na Tabela 3.

As análises de $\mathrm{DQO}_{\mathrm{S}}, \mathrm{DBO}_{5}{ }^{20^{\circ} \mathrm{C}}$, sólidos dissolvidos totais (SDT), sólidos dissolvidos fixos (SDF) e sólidos dissolvidos voláteis (SDV) descritas na Tabela 3 foram realizadas filtrando a amostra em membrana de fibra de vidro com porosidade $0,45 \mu \mathrm{m}$.

Os parâmetros operacionais avaliados foram: vazão $(\mathrm{Q})$, tempo de detenção hidráulica (TDH), carga hidráulica volumétrica (CHV), carga orgânica volumétrica (COV), carga orgânica biológica (COB), velocidade ascensional (VA) e taxa de aplicação superficial (TAS) foram calculados de acordo com Metcalf e Eddy (2003). A vazão afluente ao RAC foi controlada diariamente, sendo mensurada pelo método gravimétrico. As eficiências de remoção em termos de DQO e SS foram calculadas utilizando as Equações 12 e 13:

$\operatorname{DQO}(\%)=\left(\frac{\mathrm{DQO}_{\mathrm{T}} \text { afluente} \mathrm{DQO}_{\mathrm{F}} \text { efluente }}{\mathrm{DQO}_{\mathrm{T}} \text { afluente }}\right) \times 100$

SS $(\%)=\left(\frac{\text { SS afluente }}{\text { SS afluente }}\right) \times 100$

onde:

DQO $(\%)=$ eficiência de remoção de DQO

SS $(\%)=$ eficiência de remoção de sólidos sedimentáveis

Os parâmetros físico-químicos avaliados durante a partida ao longo do experimento, bem como as concentrações da biomassa avaliadas, foram abordados estatisticamente com três tratamentos, sendo cada tratamento representado pelas amostras A-RAC, E-RAC e E-UASB. $\mathrm{O}$ número de repetições para cada tratamento foi apresentado à direita sobrescrito ao parâmetro analisado. Para determinar se havia diferença estatística significativa entre os tratamentos, tais procedimentos foram submetidos ao teste de Tukey $(\mathrm{p}<0,05)$, sendo as diferenças ou igualdades estatísticas entre as médias demonstradas por letras ao lado da média. As análises estatísticas foram realizadas no software estatístico Sisvar (FERREIRA, 2011).

\section{RESULTADO E DISCUSSÃO}

\section{Análise dos parâmetros físico-químicos ao longo do sistema}

A partida do RAC e do UASB foi realizada e monitorada durante os meses de junho a setembro. A temperatura local média foi de $22,3^{\circ} \mathrm{C}$, tendo como máxima e mínima os valores de 34,8 e $4,4^{\circ} \mathrm{C}$, respectivamente, portanto operando na faixa mesofílica de temperatura (SPEECE, 1996; FOX \& LIM, 2010). As variações de temperatura não puderam ser controladas, uma vez que o sistema piloto foi implantado no campo e, portanto, operado em condições climáticas reais.

Os parâmetros físico-químicos avaliados durante os três meses de monitoramento da partida nos pontos A-RAC, E-RAC e E-UASB estão apresentados na Tabela 4 .

Para os parâmetros de tamponamento analisados como $\mathrm{pH}, \mathrm{AcT}$, AT, AI e AP, não foram observadas diferenças estatísticas ( $p>0,05)$ entre os valores médios das amostras A-RAC, E-RAC e E-UASB (Tabela 4). Isso mostra estatisticamente que não houve geração ou consumo dos ácidos (Ac.T) e das alcalinidades (AT, AI e AP).

Pereira, Campos e Moterani (2013) estudaram o consumo e a geração de ácidos voláteis e alcalinidade ao longo de um sistema de tratamento para ARS com biomassas adaptadas e operando em estado estacionário e concluíram que, quando se deseja obter alta eficiência de remoção de matéria orgânica com geração de alcalinidade durante o tratamento de ARS, são necessárias relações AI/AP de 1,96 para TAEs, 1,56 para reatores que operam em primeiro estágio anaeróbio e 1,44 para reatores operando em segundo estágio. Com base nas concentrações de AI e AP apresentadas na Tabela 4, nota-se que a relação AI/AP da ARS durante a partida e formação de biomassa foi, em média, de 0,52, 0,61 e 0,63, ao passar pelo TAE (A-RAC), pelo RAC (E-RAC) e pelo UASB (E-UASB), respectivamente. Portanto, a biomassa gerada por sedimentação durante a partida do RAC e do UASB ainda não estava propícia para geração de alcalinidade com valores de relações AI/AP menores que a metade dos valores ideais para cada unidade de tratamento.

Não foi observada diferença estatística $(\mathrm{p}>0,05)$ para os valores de condutividade elétrica (CE) da ARS entre as amostras A-RAC, E-RAC e E-UASB (Tabela 4). De acordo com von Sperling (2006), a CE está relacionada com a concentração de SDT da água residuária. Como pode ser observado na Tabela 4, também não houve diferença estatisticamente significativa entre as concentrações de SDT para as amostras A-RAC, E-RAC e E-UASB. 
Além dos parâmetros anteriormente mencionados, também não foi observada diferença estatisticamente significativa $(\mathrm{p}>0,05)$ para as concentrações de sólidos totais voláteis (STV), DQO $\mathrm{F}_{\mathrm{F}}$ O\&G entre as amostras A-RAC, E-RAC e E-UASB, demonstrando que não houve geração ou remoção desses parâmetros pelo sistema.

As concentrações de $\mathrm{DBO}_{5}^{20^{\circ} \mathrm{C}} \mathrm{e} \mathrm{DBO}_{5}{ }^{20^{\circ} \mathrm{C}}{ }_{\mathrm{S}}$ apresentaram diferenças estatisticamente significativas entre os valores médios de $1 \%(\mathrm{p}<0,01)$ de significância. Para as concentrações de $\mathrm{DBO}_{5} 2^{\circ 0^{\circ} \mathrm{C}}$, foram observadas remoções desse parâmetro ao passar pelo RAC, todavia a concentração de $\mathrm{DBO}_{5}{ }^{20^{\circ} \mathrm{C}}$ efluente ao RAC manteve-se estatisticamente igual à concentração efluente ao UASB, demonstrando que não houve remoção de $\mathrm{DBO}_{5}{ }^{20^{\circ} \mathrm{C}}{ }_{\mathrm{T}}$ no UASB. Já para as concentrações de $\mathrm{DBO}_{5}{ }^{20^{\circ} \mathrm{C}} \mathrm{S}$ foram observadas quedas contínuas ao passar pelo RAC e pelo UASB, comprovadas pelas diferenças estatísticas entre as médias de concentração nas três amostras avaliadas.

As concentrações de $\mathrm{DQO}_{\mathrm{T}}$ apresentaram diferenças estatisticamente significativas entre os valores médios de $1 \%(\mathrm{p}<0,01)$ de significância. Para esse parâmetro foi observado remoção ao passar pelo

Tabela 4 - Monitoramento do afluente e do efluente do reator anaeróbio de manta de lodo e do reator anaeróbio compartimentado e valores médios para cada parâmetro analisado durante a partida.

\begin{tabular}{|c|c|c|c|c|}
\hline Parâmetros físico-químicos & Estatística descritiva & A-RAC & E-RAC & E-UASB \\
\hline \multirow{2}{*}{$\mathrm{pH}^{(24)}$} & Média $\pm D P$ & $6,7 a \pm 0,5$ & $6,7 a \pm 0,3$ & 6,9a $\pm 0,2$ \\
\hline & CV (\%) & 8,0 & 4,1 & 2,5 \\
\hline \multirow{2}{*}{$\begin{array}{l}\mathrm{AcT}^{(12)} \\
\left(\mathrm{mgHCOOH} L^{-1}\right)\end{array}$} & Média $\pm D P$ & $41,7 a \pm 19,4$ & $56,5 a \pm 52,7$ & 64,1a $\pm 60,1$ \\
\hline & CV (\%) & 46,6 & 93,3 & 93,8 \\
\hline \multirow{2}{*}{$\begin{array}{l}\mathrm{Al}^{(12)} \\
\left(\mathrm{mgCaCO}_{3} \mathrm{~L}^{-1}\right)\end{array}$} & Média $\pm D P$ & $649,2 \mathrm{a} \pm 191,7$ & $669,4 a \pm 213,5$ & $794,3 a \pm 278,3$ \\
\hline & CV (\%) & 29,5 & 31,9 & 35,0 \\
\hline \multirow{2}{*}{$\begin{array}{l}\mathrm{AP}^{(12)} \\
\left(\mathrm{mgCaCO}_{3} \mathrm{~L}^{-1}\right)\end{array}$} & Média $\pm D P$ & $1239,1 a \pm 424,7$ & $1105,9 a \pm 464,0$ & $1263,2 \mathrm{a} \pm 552,1$ \\
\hline & CV (\%) & 34,3 & 42,0 & 43,8 \\
\hline \multirow{2}{*}{$\begin{array}{l}\mathrm{AT}^{(12)} \\
\left(\mathrm{mgCaCO}_{3} \mathrm{~L}^{-1}\right)\end{array}$} & Média $\pm D P$ & $1528,6 \mathrm{a} \pm 447,2$ & $1454,6 \mathrm{a} \pm 424,8$ & 1691,Oa $\pm 567,7$ \\
\hline & CV (\%) & 29,2 & 29,2 & 33,6 \\
\hline \multirow{2}{*}{$\begin{array}{l}C E^{(24)} \\
\left(d S m^{-1}\right)\end{array}$} & Média $\pm D P$ & $3,9 a \pm 1,6$ & 4,0a $\pm 1,4$ & 4,1a $\pm 1,5$ \\
\hline & CV (\%) & 41,9 & 35,7 & 37,2 \\
\hline \multirow{2}{*}{$\begin{array}{l}\mathrm{DBO}_{5}^{20^{\circ} \mathrm{C}(22)} \\
\left(\mathrm{mgO}_{2} \mathrm{~L}^{-1}\right)\end{array}$} & Média $\pm D P$ & $3008,3 a \pm 1359$ & $1934,8 b \pm 930,3$ & $1711,8 \mathrm{~b} \pm 872,8$ \\
\hline & CV (\%) & 45,2 & 48,1 & 51,0 \\
\hline \multirow{2}{*}{ 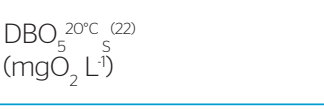 } & Média $\pm \mathrm{DP}$ & 1089,6a $\pm 206,1$ & $921,8 \mathrm{~b} \pm 243,8$ & $812,9 c \pm 223,1$ \\
\hline & CV (\%) & 18,9 & 26,4 & 27,4 \\
\hline \multirow{2}{*}{$\begin{array}{l}\mathrm{ST}^{(22)} \\
\left(\mathrm{mg} \mathrm{L^{-1 } )}\right.\end{array}$} & 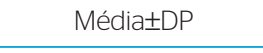 & 10633,7a $\pm 13956,4$ & $3192,8 b \pm 2998,1$ & $3639,3 \mathrm{ab} \pm 1401,4$ \\
\hline & CV (\%) & 131,2 & 82,4 & 43,9 \\
\hline \multirow{2}{*}{$\begin{array}{l}\text { STV }^{(22)} \\
\left(\mathrm{mg} \mathrm{L}^{-1}\right)\end{array}$} & Média士DP & $7389,4 a \pm 11121,5$ & 1945,0a $\pm 1135,8$ & $2484,4 a \pm 2898,6$ \\
\hline & CV (\%) & 150,5 & 58,4 & 116,8 \\
\hline \multirow{2}{*}{$\begin{array}{l}\mathrm{SDT}^{(22)} \\
\left(\mathrm{mg} \mathrm{L^{-1 } )}\right.\end{array}$} & Média士DP & $5205,2 \mathrm{a} \pm 10709,2$ & $1561,5 a \pm 352,4$ & $2240,7 \mathrm{a} \pm 2126,5$ \\
\hline & CV (\%) & 205,7 & 22,6 & 94,9 \\
\hline \multirow{2}{*}{$\begin{array}{l}\mathrm{SDV}^{(22)} \\
\left(\mathrm{mg} \mathrm{L^{-1 }}\right)\end{array}$} & 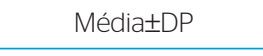 & $1049,9 a \pm 371,3$ & $737,4 b \pm 249,8$ & $820,3 a b \pm 172,6$ \\
\hline & CV (\%) & 35,4 & 33,9 & 21,0 \\
\hline \multirow{2}{*}{$\begin{array}{l}\mathrm{DQO}_{\mathrm{T}}{ }^{(22)} \\
\left(\mathrm{mgO}_{2} \mathrm{~L}^{-1}\right)\end{array}$} & Média士DP & $32294,0 a \pm 20982,2$ & $13320 b \pm 7695,6$ & $8746,1 \mathrm{~b} \pm 4921,7$ \\
\hline & CV (\%) & 65 & 57,8 & 56,3 \\
\hline \multirow{2}{*}{$\begin{array}{l}\mathrm{DQO}_{\mathrm{S}}^{(22)} \\
\left(\mathrm{mgO}_{2} \mathrm{~L}^{-1}\right)\end{array}$} & 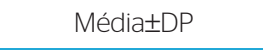 & $12918,2 a \pm 21345,2$ & $8351,5 a \pm 9463,8$ & $3872,4 a \pm 1628,0$ \\
\hline & CV (\%) & 165,2 & 113,3 & 42,0 \\
\hline \multirow{2}{*}{$\begin{array}{l}O \& G^{(12)} \\
\left(m g L^{-1}\right)\end{array}$} & Média土DP & $1947,2 a \pm 1601,6$ & $567 a \pm 269,8$ & $206,5 \mathrm{a} \pm 93,1$ \\
\hline & CV (\%) & 82,2 & 47,6 & 45,1 \\
\hline
\end{tabular}

DP: desvio padrão do conjunto amostral (mesma unidade utilizada no parâmetro); CV: coeficiente de variação do conjunto amostral (\%). 
RAC, todavia a concentração de $\mathrm{DQO}_{\mathrm{T}}$ efluente ao RAC manteve-se estatisticamente igual à concentração efluente ao UASB, demonstrando que não houve remoção desse parâmetro no UASB.

As concentrações de ST e SDV apresentaram diferenças estatisticamente significativas entre os valores médios de $5 \%(\mathrm{p}<0,05)$ de significância. Para as concentrações de ST e SDV foram observadas remoções desse parâmetro ao passar pelo RAC, todavia as concentrações médias de ST e SDV efluentes ao RAC foram estatisticamente iguais às concentrações efluentes ao UASB, demonstrando que não houve remoção desses parâmetros nesta última unidade.

Como hipótese de justificativa para os valores que não apresentaram diferença estatisticamente significativa, acredita-se que como a biomassa não estava acumulada em quantidade suficiente, nem mesmo aclimatizada, ocorreram oscilações no desempenho dos reatores, resultando em altos valores de desvio padrão e coeficiente de variação entre as amostras. Essas variações de desempenho são naturalmente esperadas durante a partida de reatores biológicos, especialmente quando a biomassa está sendo formada por meio da sedimentação de sólidos. Esse fato pode ter causado sobreposição no valor da diferença mínima significativa entre os tratamentos utilizados para comparação no teste de Tukey.

\section{Desempenho e formação de biomassa no reator anaeróbio compartimentado e no reator anaeróbio de manta de lodo}

Como pode ser observado na Tabela 3 , as concentrações afluentes de $\mathrm{DQO}_{\mathrm{T}}$ no RAC e no UASB sofreram variação. Portanto, mesmo mantendo as vazões afluentes aos reatores constantes (Tabelas 1 e 2), foram observadas variações na COV aplicada em ambas as unidades de tratamento (Figura 1).

Zeeuw (1984), estudando a partida e aclimatização de UASB, descreve que quanto menores a variação e a oscilação da carga aplicada durante a partida, mais estáveis serão os resultados de eficiência do processo estudado. Com base nessas conclusões, estima-se que as variações nos valores de COV provocaram oscilações na eficiência de remoção de DQO e SS no RAC e no UASB (Figura 1).

Desde o início do experimento foram observados altos valores de remoção de DQO no RAC e no UASB, acompanhando os altos

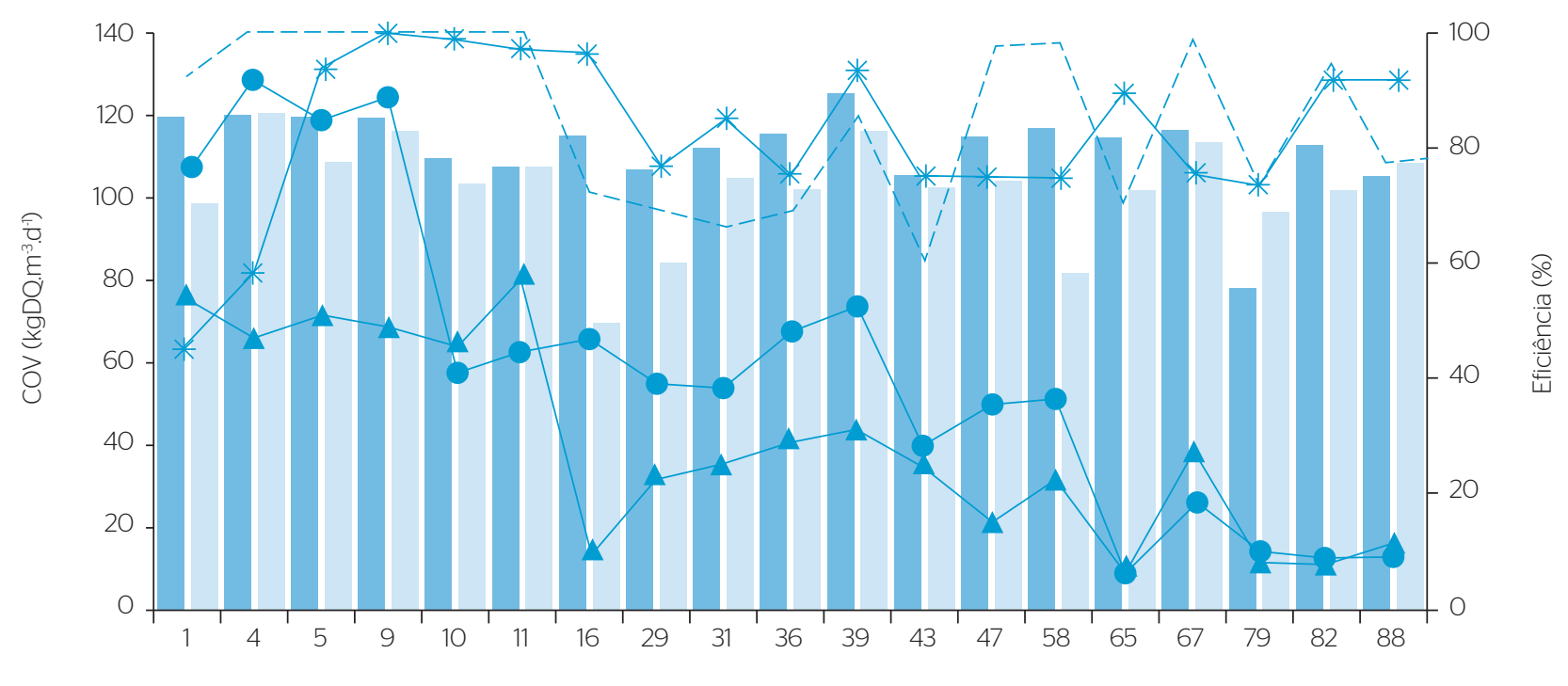

Dias de monitoramento
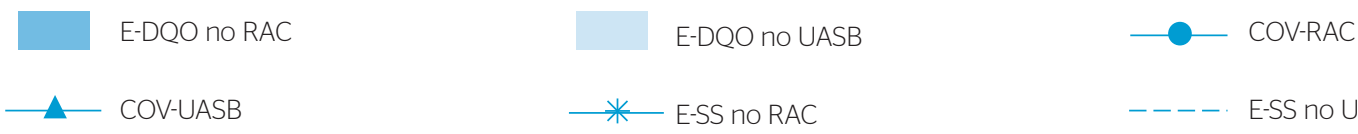

COV-UASB

COV-RAC: carga orgânica volumétrica aplicada ao reator anaeróbio compartimentado; COV-UASB: carga orgânica volumétrica aplicada ao reator anaeróbio de manta de lodo; E-DQO no RAC: eficiência de remoção de DQO no reator anaeróbio compartimentado; E-DQO no UASB: eficiência de remoção de DQO no reator anaeróbio de manta de lodo; E-SS no RAC: eficiência de remoção de sólidos sedimentáveis no reator anaeróbio compartimentado; E-SS no UASB: eficiência de remoção de sólidos sedimentáveis no reator anaeróbio de manta de lodo.

Figura 1 - Cargas orgânicas volumétricas aplicadas ao reator anaeróbio compartimentado e ao reator anaeróbio de manta de lodo e eficiências de remoção de DQO e sólidos sedimentáveis no reator anaeróbio compartimentado e no reator anaeróbio de manta de lodo. 
valores de remoção de SS. Grande parte da remoção dos SS ocorreu devido a sua decantação nos reatores, formando a biomassa desses elementos.

De acordo com Ghangrekara, Asolekarb e Joshic (2005), a COV é o parâmetro mais importante durante a partida de um reator anaeróbio, pois ela interfere no processo de formação e nas características da biomassa. Ghangrekara, Asolekarb e Joshic (2005), avaliando a partida de UASBs, também encontraram oscilações nos valores de eficiência de remoção de DQO e SS em função da variação de COV aplicada, como observado no presente trabalho.

De acordo com Chernicharo (2007), para efluentes agroindustriais com elevada concentração de matéria orgânica, a literatura reporta valores de COV extremamente elevados, da ordem de $45 \mathrm{~kg} \cdot \mathrm{m}^{-3} \cdot \mathrm{d}^{-1}$ em termos de $\mathrm{DQO}_{\mathrm{T}}$. As cargas orgânicas adotadas em reatores de escala plena tratando esgoto doméstico têm sido, via de regra, inferiores a $15 \mathrm{~kg} . \mathrm{m}^{-3} \mathrm{~d}^{-1}$ em termos de $\mathrm{DQO}_{\mathrm{T}}$ Neste trabalho foram aplicados no RAC valores de COV máximos de $130 \mathrm{~kg} \cdot \mathrm{m}^{-3} \cdot \mathrm{d}^{-1} \mathrm{em}$ termos de $\mathrm{DQO}_{\mathrm{T}}$ e no UASB valores de COV máximos de $78 \mathrm{~kg} \cdot \mathrm{m}^{-3} \cdot \mathrm{d}^{-1}$ em termos de $\mathrm{DQO}_{\mathrm{T}}$. Portanto, ficou comprovado que a utilização, durante a partida, de valores de COV bem maiores que os citados por Chernicharo (2007) não prejudicou o processo, mesmo objetivando a formação de biomassa por meio da sedimentação dos SS presentes na própria ARS.

Abreu Neto e Oliveira (2009) avaliaram o efeito das ARSs com concentrações médias de sólidos suspensos totais variando de 4.591 a $13.000 \mathrm{mg} . \mathrm{L}^{-1}$, no desempenho de RACs e UASBs em escala piloto, instalados em série, com volumes de 530 e $120 \mathrm{~L}$, respectivamente. O TDH aplicado ao RAC foi de 60, 36 e 24 h e de 13,6, 8,2 e 5,4h no UASB. As eficiências médias de remoção de $\mathrm{DQO}_{\mathrm{T}}$ variaram de 69 a $84 \%$ no RAC e de 39 a 58\% no UASB, resultando em valores médios de 87 a 94\% para o sistema de tratamento anaeróbio em 2 estágios. As COVs no RAC, em termos de $\mathrm{DQO}_{\mathrm{T}}$, variaram na faixa de $11,5 \mathrm{a}$ $18,0 \mathrm{~kg} \cdot \mathrm{m}^{-3} \cdot \mathrm{d}^{-1}$, e no UASB, de 4,2 a 13,4 kg. $\mathrm{m}^{-3} \cdot \mathrm{d}^{-1}$.

Pereira et al. (2011), tratando ARS em sistema de RAC e UASB operando em série, com TDHs de 15,4 e 9,7 h, CHs de 1,57 e 2,5 $\mathrm{m}^{3} \cdot \mathrm{m}^{-3} \cdot \mathrm{d}^{-1}$, COVs de 4,46 e $1,77 \mathrm{~kg} . \mathrm{m}^{-3} \cdot \mathrm{d}^{-1}$ em termos de $\mathrm{DQO}_{\mathrm{T}}$, respectivamente, observaram eficiências médias de remoção de $\mathrm{DBO}_{5}{ }^{20^{\circ} \mathrm{C}}$ e $\mathrm{DQO}_{\mathrm{T}}$ de 91,50 e $85,24 \%$, respectivamente. Comparando os dados apresentados no que diz respeito à eficiência de remoção de $\mathrm{DBO}_{5}{ }^{20^{\circ} \mathrm{C}} \mathrm{e} \mathrm{DQO}_{\mathrm{T}}$ e aos parâmetros operacionais aplicados, foi constatado que os reatores operaram com valores de COV elevados, e, consequentemente, TDH inferior àqueles descritos na literatura para sistemas similares, $\mathrm{e}$ obtiveram eficiências de remoção de $\mathrm{DBO}_{5}^{20^{\circ} \mathrm{C}} \mathrm{T}_{\mathrm{T}}$ e $\mathrm{DQO}_{\mathrm{T}}$ relativamente altas, quando comparadas às apresentadas na literatura. Todavia, deve-se levar em consideração que os experimentos avaliados estavam operando com biomassa aclimatizada, enquanto neste trabalho foi avaliada a formação da biomassa por sedimentação, obtendo, assim, oscilações nos valores de eficiência em função da ausência de lodo aclimatizado no interior dos reatores. Constata-se, pelas eficiências obtidas durante a partida, que se levarmos em consideração os altos valores de COV aplicados, com a ausência de biomassa aclimatizada no interior das referidas unidades, estas operaram com eficiências relativamente elevadas.

Na Figura 1 foram mostradas as eficiências de remoção de SS. Esses sólidos propiciaram a formação da biomassa no RAC (Figura 2) e no UASB (Figura 3).

Nas Figuras 2 e 3 estão apresentadas as quantidades de biomassa formada ao longo do tempo, no RAC e no UASB, em relação aos valores de $\mathrm{COB}$ aplicados aos reatores. Como pode ser observado, os valores de COB aplicados no RAC (Figura 2) e no UASB (Figura 3) iniciaram máximos devido à baixa quantidade de biomassa existente no $1^{\circ}$ dia de avaliação do perfil de lodo, que ocorreu 20 dias após o início do funcionamento. De acordo com a equação utilizada para calcular a COB, descrita em Metcalf e Eddy (2003), para a mesma carga o aumento da massa de lodo diminuiu em função do valor da $\mathrm{COB}$ aplicada. Isso justifica o comportamento observado no RAC (Figura 2) e UASB (Figura 3), mostrando que com o passar do tempo ocorreu aumento na massa de lodo e diminuição dos valores de COB aplicados. Além das oscilações nos valores de COB aplicados foram observadas as diferenças estatísticas entre os valores concentração de biomassa de cada amostrador, durante a primeira e última coleta, demonstrando que o processo de formação de biomassa não foi constante ou estritamente crescente em quantidade de massa. A Tabela 5 apresenta o perfil de biomassa ao longo da altura do RAC e do UASB.

Como pode ser observado na Tabela 5, as concentrações médias de STV obtidas nos amostradores foram observadas diferentes estatisticamente a $1 \%(\mathrm{p}<0,01)$, tanto para a amostragem realizada no primeiro dia de avaliação, quanto para a amostragem realizada no último dia. Portanto, conclui-se que desde a primeira amostragem feita sete dias após o início da partida já havia diferença de concentração de sólidos voláteis entre os amostradores. As mesmas diferenças estatísticas entre os amostradores permaneceram até a última avaliação, como valores de concentração, em módulo, maiores que os obtidos na primeira avaliação.

Como os valores de concentração de STV obtidos na última avaliação foram estatisticamente diferentes $(\mathrm{p}<0,01)$ dos obtidos na primeira avaliação e em módulo, e maiores que os valores obtidos na primeira avaliação, conclui-se que houve um aumento na concentração de STV ao longo da partida no RAC e no UASB. Isso comprovou que por meio do processo de decantação dos SS da própria ARS foi possível formar biomassa em quantidade suficiente capaz de permitir a partida das referidas unidades (Figuras 2 e 3). A concentração de STV relativos à biomassa no RAC e no UASB pode também ser visualizada na Tabela 5 . 


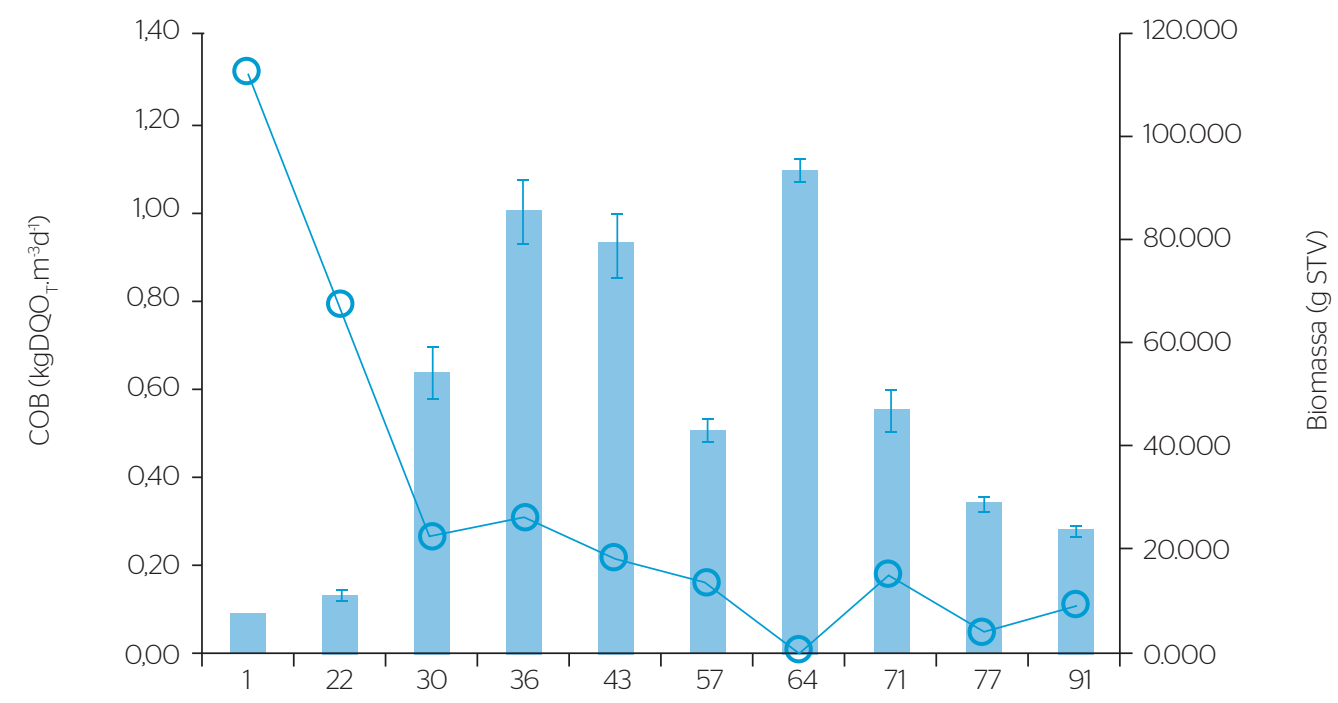

Dias de monitoramento

COB-RAC: cargas orgânicas biológicas aplicadas ao reator anaeróbio compartimentado; DQO: concentração de DQO total (solúvel + particulada) da água residuária de suinocultura afluente do reator anaeróbio compartimentado; STV: concentração de sólidos totais voláteis na biomassa do reator anaeróbio compartimentado.

Figura 2 - Cargas orgânicas biológicas aplicadas ao reator anaeróbio compartimentado e biomassa formada ao longo do período de partida devido à sedimentação dos sólidos sedimentáveis da própria água residuária de suinocultura.

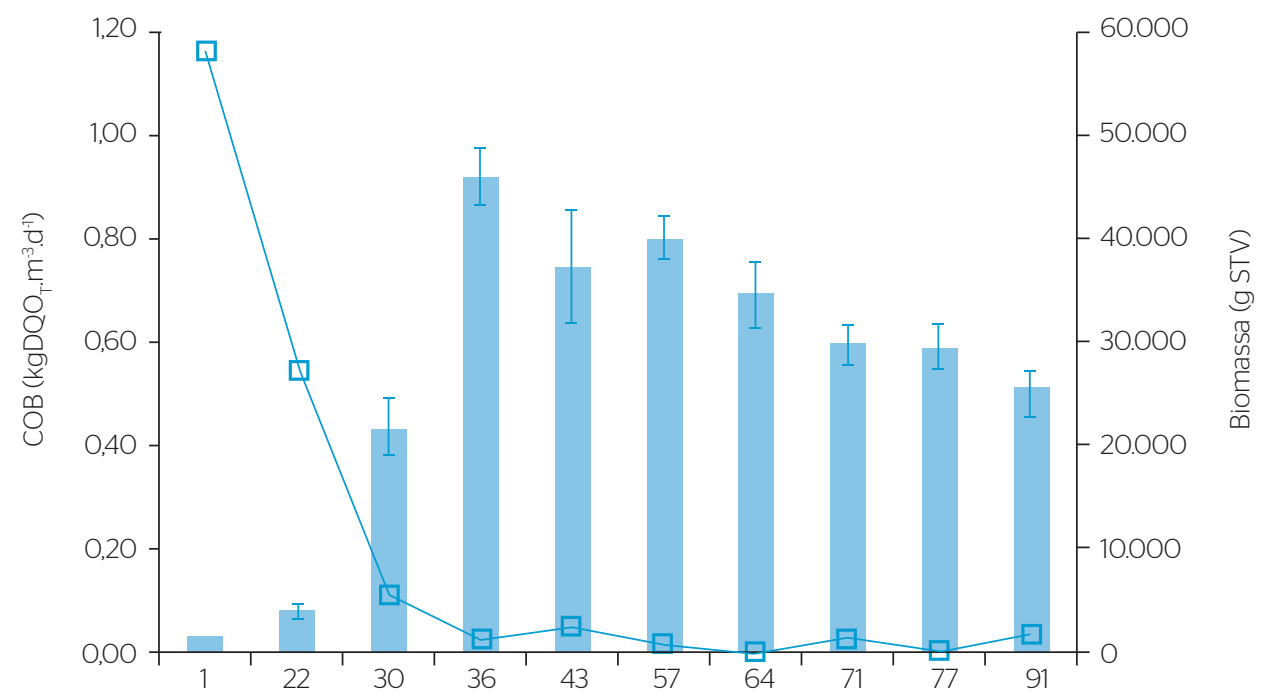

Dias de monitoramento

COB-UASB: cargas orgânicas biológicas aplicadas ao reator anaeróbio de manta de lodo; DQO: concentração de DQO total (solúvel+particulada) da água residuária de suinocultura afluente do reator anaeróbio de manta de lodo; STV: concentração de sólidos totais voláteis na biomassa do reator anaeróbio de manta de lodo.

Figura 3 - Cargas orgânicas biológicas aplicadas ao reator anaeróbio de manta de lodo e biomassa formada ao longo do período de partida devido à sedimentação dos sólidos sedimentáveis da própria água residuária de suinocultura. 
Ghangrekara, Asolekarb e Joshic (2005), avaliando a partida de um UASB com biomassa inoculada, observaram que para formação de uma biomassa granulada foi necessário controlar os parâmetros de COV e COB aplicados, sendo estes parâmetros os fatores mais importantes no processo de granulação do lodo durante a partida. Os autores sugerem taxas de 2,0 e 4,5 kg.m ${ }^{-3} \cdot \mathrm{d}^{-1} \mathrm{em}$ termos de $\mathrm{DQO}_{\mathrm{T}}$ para a COV e valores de COB entre 0,1 e 0,25 $\mathrm{kgDQO}_{\mathrm{T}} \cdot \mathrm{kgSTV}^{-1}$. $\mathrm{d}^{-1}$. A partida e a formação de biomassa no RAC e no UASB neste trabalho utilizaram, respectivamente, valores de COV aproximadamente 30 e 20 vezes maiores que os sugeridos por Ghangrekara, Asolekarb e Joshic (2005). Já as COBs aplicadas ao RAC e ao UASB tiveram valores 14 e 12 vezes maiores, respectivamente, durante o início da partida, mas durante o processo de formação da biomassa as mesmas ficaram dentro da faixa ótima descrita por Ghangrekara, Asolekarb e Joshic (2005).

Com base nos resultados apresentados nas Figuras 2 e 3 e na Tabela 5 é possível afirmar que os parâmetros de sedimentação empregados no processo de partida do RAC $\left(\mathrm{TDH}=14,5 \mathrm{~h} ; \mathrm{TAS}=4,6 \mathrm{~m}^{3} \cdot \mathrm{m}^{-3} \cdot \mathrm{d}^{-1} ; \mathrm{IVL}=0,75 \mathrm{~m}^{3}\right.$ de SS $\mathrm{m}^{-3}$ de reator; $\mathrm{VA}=0,19 \mathrm{~m} \cdot \mathrm{h}^{-1} ; \mathrm{CH}=1,7 \mathrm{~m}^{3} \cdot \mathrm{m}^{-3} \cdot \mathrm{d}^{-1}$, operando com concentração de SS afluente de 113 mL. $\mathrm{L}^{-1}$ ) e do UASB (TDH=9,06 h; TAS $=5,67 \mathrm{~m}^{3} \cdot \mathrm{m}^{-3} \cdot \mathrm{d}^{-1} ; \mathrm{IVL}=1,53 \mathrm{~m}^{3}$ de SS $\mathrm{m}^{-3}$ de reator; VA=0,24 m.h $\mathrm{h}^{-1}$; $\mathrm{CHV}=2,7 \mathrm{~m}^{3} \cdot \mathrm{m}^{-3} \cdot \mathrm{d}^{-1}$, operando com concentração de SS afluente de 84,5 mL.L $\left.\mathrm{L}^{-1}\right)$ mostraram ser satisfatórios para a obtenção de biomassa anaeróbia com boa concentração de STV, mesmo com valores de cargas $\mathrm{COV}$ e $\mathrm{COB}$ muito maiores que os indicados na literatura pesquisada.
Após 90 dias de avaliação houve elevada estabilização no processo de formação da biomassa por decantação dos SS, e os valores de eficiência variaram no máximo $5 \%$ entre si, indicando que os reatores haviam entrado em estado estacionário. Após a partida, a fase estacionária foi monitorada. Durante o estado estacionário foram realizados a caracterização hidrodinâmica dos reatores e o levantamento dos parâmetros cinéticos do lodo, com a caracterização microbiológica, utilizando técnicas de microscopia eletrônica e estudos sobre a influência de choques orgânicos e hidráulicos no desempenho do RAC e do UASB. Os referidos estudos estão apresentados em Matangue (2011).

Com base nos dados apresentados por Matangue (2011), a biomassa gerada por meio da decantação dos SS da própria ARS formou grânulos ricos em bactérias do tipo cocos e filamentosas principalmente nas amostras coletadas no primeiro amostrador. Além disso, o processo demonstrou alta eficiência na remoção de DQO e $\mathrm{DBO}_{5}{ }^{20^{\circ} \mathrm{C}}$.

Ressalva-se ainda que as altas cargas e os baixos TDHs aplicados contribuíram para celeridade na formação da biomassa, satisfazendo, assim, a expectativa inicial de mostrar a possibilidade de o sistema de tratamento partir sem ter custos adicionais referentes a transporte, compra e bombeamento do lodo para inoculação e partida. Outra observação importante foi que o estudo demonstrou que a biomassa formada com o próprio efluente permite a formação de um lodo bem mais aclimatizado do que os de inoculação com

Tabela 5 - Perfil de sólidos totais voláteis da biomassa no reator anaeróbio compartimentado (compartimentos 1, 2 e 3) e no reator anaeróbio de manta de lodo: comparação estatística entre os valores das concentrações nos amostradores ao longo da altura dos reatores e entre os valores médios no primeiro e último dia de avaliação do sistema.

\begin{tabular}{|c|c|c|c|c|c|}
\hline \multicolumn{3}{|c|}{ RAC - Compartimento 1} & \multicolumn{3}{|c|}{ RAC - Compartimento 2} \\
\hline $\mathrm{H}(\mathrm{cm})$ & $\begin{array}{l}\text { Primeira amostragem } \\
\quad\left(\text { mgSTV } L^{-1}\right)\end{array}$ & $\begin{array}{l}\text { Última amostragem } \\
\quad(\text { mgSTV L'1) }\end{array}$ & $\mathrm{H}(\mathrm{cm})$ & $\begin{array}{l}\text { Primeira amostragem } \\
\quad(\text { mgSTV L-1) }\end{array}$ & $\begin{array}{l}\text { Última amostragem } \\
\quad\left(\operatorname{mgSTV} \mathrm{L}^{-1}\right)\end{array}$ \\
\hline 33 & $2328,2 \mathrm{a} A \pm 0,2$ & $5573,2 \mathrm{a} B \pm 0,2$ & 34 & 1740,0a A $\pm 0,1$ & $8132,8 \mathrm{a} \mathrm{B} \pm 0,7$ \\
\hline 71 & 2259,6b A $\pm 0,5$ & 6290,6b B $\pm 0,6$ & 75 & 2710,0b A $\pm 0,1$ & 4813,3b B $\pm 0,2$ \\
\hline 119 & $2864,6 \mathrm{c} A \pm 0,6$ & $6174,6 \mathrm{c}$ B $\pm 0,5$ & 120 & $1084,6 \mathrm{c} A \pm 0,6$ & 4866,7c B $\pm 0,5$ \\
\hline 168 & $3654,6 \mathrm{~d} A \pm 0,6$ & $3493,3 d \mathrm{~B} \pm 0,2$ & 168 & $1854,6 \mathrm{~d} A \pm 0,6$ & $3674,9 d \mathrm{~d} \pm 0,1$ \\
\hline \multicolumn{3}{|c|}{ RAC - Compartimento 3} & \multicolumn{3}{|c|}{ UASB } \\
\hline $\mathrm{H}(\mathrm{cm})$ & $\begin{array}{l}\text { Primeira amostragem } \\
\quad(\text { mgSTV L-1) }\end{array}$ & $\begin{array}{l}\text { Última amostragem } \\
\quad(\text { mgSTV L-1) }\end{array}$ & $\mathrm{H}(\mathrm{cm})$ & $\begin{array}{l}\text { Primeira amostragem } \\
\left(\text { (mgSTV } L^{-1}\right)\end{array}$ & $\begin{array}{l}\text { Última amostragem } \\
\left(\operatorname{mgSTV} \mathrm{L}^{-1}\right)\end{array}$ \\
\hline 35 & 1420,Oa A $\pm 0,1$ & 13369,8a B士0,3 & 16 & 1175,0 a $\mathrm{A} \pm 0,1$ & 36237,3a B $\pm 0,3$ \\
\hline 66 & $1093,2 b$ A $\pm 0,2$ & 4438,2b B $\pm 0,2$ & 42 & $1140,0 b \mathrm{~A} \pm 0,2$ & 25070,0b B $\pm 0,1$ \\
\hline 122 & $1200,0 c \mathrm{~A} \pm 0,1$ & 8034,9 c B $\pm 0,1$ & 58 & $1149,8 \mathrm{c} A \pm 0,3$ & $16252,5 \mathrm{c} \mathrm{B} \pm 0,5$ \\
\hline 168 & $1374,9 d A \pm 0,1$ & 3895,Od B $\pm 0,1$ & 77 & $1153,2 d A \pm 0,2$ & $19223,2 d \mathrm{~B} \pm 0,2$ \\
\hline
\end{tabular}

As amostragens foram feitas em triplicata e os resultados utilizados para determinação do valor médio de concentração, sendo a primeira amostragem sete dias após a partida e a última amostragem 95 dias após a partida.

H: altura do amostrador em relação à base do reator (cm); RAC: reator anaeróbio compartimentado; UASB: reator anaeróbio de manta de lodo. Letras minúsculas representam comparação entre os amostradores, e letras maiúsculas, comparação entre as médias do mesmo amostrador no primeiro e último dia de avaliação. 
lodo importado de outros sistemas de tratamento. O lodo gerado por meio do próprio efluente propicia uma biomassa aclimatizada às condições de antibióticos e outros compostos bactericidas e/ou bacteriostáticos presentes no próprio dejeto, tornando o lodo com elevada resistência.

\section{CONCLUSÕES}

É possível formar biomassa anaeróbia em reatores operando no tratamento de ARS com concentrações mínimas de SS de 112 mL.L-1 e sólidos totais voláteis de $7.389 \mathrm{mg} . \mathrm{L}^{-1}$. Portanto, nesses casos não é necessária a inoculação de biomassa para partida dos reatores anaeróbios de alta taxa, como os pesquisados neste trabalho.
O processo de formação de biomassa por sedimentação, utilizando os parâmetros operacionais descritos neste trabalho, pode ser aplicado aos sistemas construídos em escalas piloto e plena, instalados nas mais longínquas distâncias de outras estações ou fontes para coleta de biomassa devido à facilidade operacional.

O lodo cultivado possui elevado grau de aclimatização e foi capaz de formar grânulos com elevada capacidade de sedimentação.

A eficiência de remoção da matéria orgânica se mostrou satisfatória e, caso haja necessidade de se elevar o nível de remoção, unidades de pós-tratamento deverão ser utilizadas.

O sistema operando sob condições de partida com elevadas cargas orgânicas apresentou relações AI/AP de 0,52, 0,61 e 0,63 para as unidades TAE, RAC e UASB, respectivamente.

\section{REFERÊNCIAS}

ABREU NETO, M.S. \& OLIVEIRA, R.A. (2009) Remoção de matéria orgânica, nutrientes e coliformes no processo anaeróbio em dois estágios (reator compartimentado seguido de reator UASB) para o tratamento de águas residuárias de suinocultura. Engenharia Agrícola, v. 29, n. 2, p. 148-161.

APHA - American Public Health Association; AWWA - American Water Works Association; WEF - Water Environment Federation. (2005) Standard methods for the examination of water and wastewater. $21^{\text {th }}$. ed. Washington. D.C.: APHA/AWWA/WEF [s.n. ].

CAMPOS, C.M.M.; PRADO, M.A.C.; PEREIRA, E.L. (2014) Kinetic parameters of biomass growth in a UASB reactor treating wastewater from coffee wet processing (WCWP). Revista Ambiente \& Água, v. 9, n. 4, p. 577-592.

CHERNICHARO, C.A.L. (2007) Reatores anaeróbios: princípios do tratamento biológico de águas residuárias. Belo Horizonte: Polytécnica. 379p

FERREIRA, D.F. (2011) Sisvar: a computer statistical analysis system. Ciência e Agrotecnologia, v. 35, n. 6, p. 1039-1042

FOX, P.E. \& LIM, J.S. (2010) A kinetic evaluation of anaerobic treatment of swine wastewater at two temperatures in a temperate climate zone. Bioresource Technology, v. 87, n. 42, p. 8878-8886.

GHANGREKARA, M.M.; ASOLEKARB, S.R.; JOSHIC, S.G. (2005) Characteristics of sludge developed under different loading conditions during UASB reactor start-up and granulation. Water Research, v. 39, n. 3, p. 1123-1133.

KIM, W.; HWANG, K.; SHIN, S.G.; LEE, S. HWANG, S. (2009) Effect of high temperature on bacterial community dynamics in anaerobic acidogenesis using mesophilic sludge inoculum. Bioresource Technology, v. 101, suplementar, S17-S22.
MATANGUE, M.T.A. (2011) Hidrodinâmica e cinética de reatores anaeróbio compartimentado (RAC) e de manta de lodo (UASB) operados em série. Dissertação (Mestrado em Engenharia Agrícola) Universidade Federal de Lavras. 395p.

METCALF \& EDDY. (2003) Waste water engineering: treatment, disposal and reuse. $4^{\text {th }}$ ed. New Delhi: Tata McGraw-Hill, 1334p.

MOTTERAN, F.; PEREIRA, E.L.; CAMPOS, C.M.M. (2O13a) Characterization of an acidification and equalization tank (AET) operating as a primary treatment of swine liquid effluent. Brazilian Archives of Biology and Technology, v. 56, n. 3, p. 485-494.

MOTTERAN, F.; PEREIRA, E.L.; CAMPOS, C.M.M. (2013b) The behaviour of an anaerobic baffled reactor (ABR) as the first stage in the biological treatment of hog farming effluents. Brazilian Journal of Chemical Engineering, v. 30, n. 2, p. 299-310.

OLIVEIRA, R.A. \& SANTANA, A.M. (2011) Tratamento de águas residuárias de suinocultura em reatores anaeróbios de fluxo ascendente com manta de lodo (UASB) em dois estágios seguidos de reator operado em batelada sequencial (RBS). Revista Engenharia Agrícola, v. 31, n. 3, p. 178-192.

PEREIRA, E.L.; CAMPOS, C.M.M.; BOAS, R.B.V; NEVES, C.S. (2O15) Use of Photolysis (Artificial Radiation-UV) as an Advanced Oxidation Process (AOP) for polishing swine wastewater treated in anaerobic reactors. Engenharia na Agricultura, v. 23, n. 3, p. 228-240.

PEREIRA, E.L.; CAMPOS, C.M.M.; MOTERANI, F. (2009) Efeitos do pH, acidez e alcalinidade na microbiota de um reator anaeróbio de manta de lodo (UASB) tratando efluentes de suinocultura. Revista Ambiente \& Água, v. 4, n. 3, p. 157-168.

PEREIRA, E.L.; CAMPOS, C.M.M.; MOTERANI, F. (2010a) Evaluation of physical-chemical performance of an UASB reactor in removing pollutants of pig wastewater. Revista Ambiente \& Água, v. 5, n. 1, p. 79-88 
PEREIRA, E.L.; CAMPOS, C.M.M.; MOTERANI, F. (2010b) Physicalchemical and operational performance of an anaerobic baffled reactor (ABR) treating swine wastewater. Acta Scientiarum. Technology, v. 32, n. 4 , p. 399-405.

PEREIRA, E.L.; CAMPOS, C.M.M.; MOTERANI, F. (2013) Physicochemical study of $\mathrm{pH}$, alkalinity and total acidity in a system composed of Anaerobic Baffled Reactor (ABR) in series with Upflow Anaerobic Sludge Blanket reactor (UASB) in the treatment of pig farming wastewater. Acta Scientiarum. Technology, v. 35, n. 3. p. 477-483.

PEREIRA, E.L.; CAMPOS, C.M.M.; MOTERANI, F.; OLIVEIRA NETO, A.M. (2011) Eficiência de um sistema de reatores anaeróbios no tratamento de efluentes líquidos de suinocultura. Acta Scientiarum. Technology, v. 33, n. 3, p. 287-293.

PEREIRA, E.L.; CAMPOS, C.M.M.; NEVES, C.S.; BOAS, R.B.V. (2014) Sterilization of swine wastewater treated by anaerobic reactors using UV photo-reactors. Acta Scientiarum. Technology, v. 36, n. 4, p. 607-615.
RIPLEY, L.E.; BOYLE, W.C.; CONVERSE, J.C. (1986) Improved alkalimetric monitoring for anaerobic digestion of high-strength wastes. Journal Water Pollution Control Federation, v. 58, n. 5, p. 406-411.

SPEECE, R.E. (1996) Anaerobic biotechnology for industrial wastewaters. Tennessee: Vanderbilt University: Archae Pr. 389p.

VAN HAANDEL, A.C.; KATO, M.T; CAVALCANTI, P.F.F. (2006) Anaerobic Reactor Design Concepts for the Treatment of Domestic Wastewater Reviews in Environmental Science and Bio-Technology, v. 5, n. 1, p. 21-38.

VAN HAANDEL, A.C. \& LETTINGA, G. (1994) Anaerobic sewage treatment: a pratical guide for regions with a hot climate. Chichester John Wiley and Sons. 226p.

VON SPERLING, M. (1996) Introdução a qualidade das águas e ao tratamento de esgoto. v. 1. DESA: UFMG. 243p.

ZEEUW, W.J.D. (1984) Acclimatization of anaerobic sludge for UASBreactor start-up. Agricultural University of Wageningen. 156p. 